Open Access

\title{
Gene expression meta-analysis reveals immune response convergence on the IFNY-STAT1-IRF1 axis and adaptive immune resistance mechanisms in lymphoma
}

\author{
Matthew A. Care ${ }^{1,2}$, David R. Westhead ${ }^{2}$ and Reuben M. Tooze ${ }^{1 *}$
}

\begin{abstract}
Background: Cancers adapt to immune-surveillance through evasion. Immune responses against carcinoma and melanoma converge on cytotoxic effectors and IFNY-STAT1-IRF1 signalling. Local IFN-driven immune checkpoint expression can mediate feedback inhibition and adaptive immune resistance. Whether such coupled immune polarization and adaptive resistance is generalisable to lymphoid malignancies is incompletely defined. The host response in diffuse large B-cell lymphoma (DLBCL), the commonest aggressive lymphoid malignancy, provides an empirical model.
\end{abstract}

Methods: Using ten publicly available gene expression data sets encompassing 2030 cases we explore the nature of host response in DLBCL. Starting from the "cell of origin" paradigm for DLBCL classification, we use the consistency of differential expression to define polarized patterns of immune response genes in $\mathrm{DLBCL}$, and derive a linear classifier of immune response gene expression. We validate and extend the results in an approach independent of "cell of origin" classification based on gene expression correlations across all data sets.

Results: T-cell and cytotoxic gene expression with polarization along the IFNY-STAT1-IRF1 axis provides a defining feature of the immune response in $\mathrm{DLBCL}$. This response is associated with improved outcome, particularly in the germinal centre B-cell subsets of DLBCL. Analysis of gene correlations across all data sets, independent of "cell of origin" class, demonstrates a consistent association with a hierarchy of immune-regulatory gene expression that places IDO1, LAG3 and FGL2 ahead of PD1-ligands CD274 and PDCD1LG2.

Conclusion: Immune responses in DLBCL converge onto the IFNY-STAT1-IRF1 axis and link to diverse potential mediators of adaptive immune resistance identifying future therapeutic targets.

\section{Background}

Emergence of clinically detectable malignant disease is associated with escape from tumour immune surveillance [1]. Two principal mechanisms may operate: on the one hand the immune systems loses the ability to detect the neoplastic population through changes in antigen presentation or editing of the antigen receptor repertoire; on the other hand initially effective immune responses may be rendered ineffective through

\footnotetext{
* Correspondence: r.tooze@leeds.ac.uk

'Section of Experimental Haematology, Wellcome Trust Brenner Building, Leeds Institute of Cancer and Pathology, University of Leeds, Leeds LS9 7TF, UK

Full list of author information is available at the end of the article
}

development of an immune suppressive environment [2]. In the latter scenario, local expression of immune checkpoint components can be viewed as subversion of a physiological mechanism, which acts during chronic infections to balance effective immunity with immunemediated tissue damage [3].

In a range of cancers the density, location and functional polarization of tumour infiltrating lymphocytes are of prognostic value [4], providing evidence that the nature of immune evasion remains of importance after clinical detection. This is particularly relevant in the context of novel therapeutic strategies aimed at re-invigorating the "exhausted" anti-tumour immune response through immune checkpoint blockade $[5,6]$. Gene expression 
analysis of bulk tumour tissue integrates expression profiles from multiple cellular sources, often allowing global assessment of the predominant vector of functional immune polarization. A paradigm has been proposed in which cancer-associated immune responses converge on a common "immunologic constant of rejection" characterized by a pattern of cytotoxic and T-cell immune responses and a dominant IFN $\gamma$-STAT1-IRF1 signalling axis $[4,7]$. Linking the polarized pattern of interferon (IFN) $\gamma$-driven immune responses to the expression of immune checkpoints is the concept of "adaptive immune resistance" [5, 8]. In this model IFNY signalling drives local feedback inhibition through the transcriptional regulation of ligands for the inhibitory receptor PD1 $[5,8]$. The common association between cytotoxic responses and expression of IFN signatures and potential mediators of adaptive immune resistance has been further supported by analysis of solid tumour gene expression data from The Cancer Genome Atlas [9]. Importantly, such feedback may be mediated both at the immediate interface between tumour cell and cytotoxic lymphocyte, and by the establishment of a wider immune suppressive milieu in the tumour microenvironment.

The combination of convergent IFN-polarized immune responses $[4,7]$, coupled to IFN-driven adaptive immune resistance $[5,8]$, provides a powerful model with which to explain common pathologic associations in carcinoma and melanoma. The recent success of therapies targeting CTLA4 and PD1 immune checkpoints [10-12], combined with an extended range of other therapeutic options [6], means that evidence to support prioritization of therapeutic combinations in different tumour settings is required. Lymphoma, which comprises immune system malignancies, provides an instance in which these pathways are incompletely studied. Classical Hodgkin lymphoma is the archetype in which host response elements dominate to the point of obscuring the neoplastic B-cell clone [13], and in classical Hodgkin lymphoma PD1 pathway blockade has recently been described as a promising therapeutic approach [14]. Diffuse large B-cell lymphoma (DLBCL) is the commonest form of nodal lymphoma in the western world and represents an aggressive malignancy that frequently remains incurable. It is well established that this lymphoma type is associated with a varied extent of host response at diagnosis, which can include elements of IFN signalling [15]. Since several large data sets are publicly available [15-25], this malignancy represents an empirical human model in which to test the association between immune polarization and adaptive immune resistance mechanisms.

The "cell of origin" (COO) classification provides the dominant paradigm for our current understanding of DLBCL [24, 26]. This classification relates the gene expression profiles in DLBCL to those of germinal centre $B$ cells $(\mathrm{GCBs})$ or activated $\mathrm{B}$ cells $(\mathrm{ABCs})$, the latter representing the initial stage of $\mathrm{B}$-cell terminal differentiation to plasma cells. Although the COO classification allows the division of DLBCL based on expression of a restricted set of classifier genes into the two principal classes [24], a subset of cases show patterns of classifier gene expression that do not allow confident assignment to either GCB or ABC subsets. Such cases are referred to as "type 3" [24, 26], or "unclassified" [27, 28]. To avoid ambiguity we refer to these cases as COOunclassified DLBCL in the following. In a parallel "consensus cluster" classification developed by Monti et al. [15], it was shown that DLBCL could be divided into three categories characterized by preferential expression of genes linked to proliferation and B-cell receptor signalling, metabolic oxidative phosphorylation, or host response. The latter included multiple elements attributable to components of the immune system and supporting stromal cell types. It was noted that a greater proportion of COO-unclassified DLBCL belonged to the host/ immune response cluster, which had increased numbers of intra-tumoral $\mathrm{T}$ cells and macrophages and a relative decrease in neoplastic B cells [15].

We reasoned that the potential association of COOunclassified DLBCL with intense host responses provided a starting point for a meta-analysis of immune response elements in DLBCL. In originating from a prevailing paradigm this provided a wider biological and clinical context. Furthermore, by asking whether evidence supporting a common polarized immune response could be discovered from within the construct of the COO paradigm, we sought to avoid bias that might have arisen by focusing ab initio on components of the polarized immune response or immune checkpoints. With this approach we identify a distinct signature characterised by a pattern of cytotoxic T-cell and IFN $\gamma$-polarized immune response genes as a dominant pattern across ten DLBCL data sets encompassing 2030 cases. Using components of this polarized pattern we then explore the immune context of DLBCL in a fashion independent of $\mathrm{COO}$ class. We demonstrate the strong association with an IFNYSTAT1-IRF1 axis and an expression hierarchy of immune checkpoints/modulators, consistent with adaptive immune resistance as a common feature operating in DLBCL.

\section{Methods}

Data sets

Ten DLBCL data sets were downloaded from the Gene Expression Omnibus (GEO) [29] [GEO:GSE4475, GSE 10846, GSE12195, GSE19246, GSE22470, GSE22895, GS E31312, GSE32918, GSE34171 and elsewhere [15-25]. GSE10846 was split according to treatment groups 
(CHOP [cyclophosphamide, doxorubicin hydrochloride (hydroxydaunomycin), vincristine sulfate (Oncovin), prednisone]/R-CHOP [rituximab-CHOP]), which were treated independently for analysis, thus giving a total of 11 data sets.

\section{Normalisation and re-annotation of data}

For each data set the probes were re-annotated with the latest version of HUGO Gene Nomenclature Committee (HGNC)-approved symbols [30]. The complete HGNC list was downloaded (on 1 October 2014). Each probe was re-annotated to the latest approved symbol if an unambiguous mapping (i.e. single symbol mapping to approved symbol) could be determined, else the original gene name was maintained.

Each data set was quantile normalised using the $R$ Limma package [31]. The probes for each gene were merged by taking the median value for probe sets with a Pearson correlation $\geq 0.2$ and the maximum value for those with a correlation $<0.2[15]$.

\section{COO classifications}

We used the $\mathrm{COO}$ classifications assigned by the DLBCL automatic classifier (DAC) classifier in our previous work [32].

\section{Meta-profile generation}

See Additional file 1 for an outline of meta-profile generation using $\mathrm{COO}$ classification.

For each of the 11 data sets a linear model was fitted to the gene expression data using the R Limma package. Differentially expressed genes between the three classes were gauged using the Limma empirical Bayes statistic module, adjusting for multiple testing using Benjamini and Hochberg correction.

The absolute fold changes for all genes per data set were normalised between 0 and 1 . The results were merged across data sets retaining only genes with an adjusted $p$ value (false discovery rate, $\mathrm{FDR}<0.05$. A meta-profile was created for each contrast (e.g. upABC_GCB) by retaining all genes differentially expressed in six or more data sets. These were then used to draw Wordles [33] with each gene's score set to $\left(\right.$ NumDataSets $\left.{ }^{3}\right) \times$ NormalisedFoldChange.

\section{Signature enrichment analysis}

A data set of 14,104 gene signatures was created by merging signatures downloaded from SignatureDB [34], MSigDB v.4 (MSigDB C1-C7) [35], Gene Signature Database v.4 (GeneSigDB) [36] and the work of Monti et al. [15] and others [37-40]. Enrichment of metaprofiles against signatures was assessed using a hypergeometric test, where the draw is the meta-profile genes, the successes are the signature genes and the population is the genes present on the platform.

\section{Gene ontology analysis}

Meta-profile gene lists were assessed for gene ontology (GO) enrichment using the Cytoscape BiNGO tool [41]. GO and annotation files were downloaded from [42] (13 June 2014). The background reference was set to a nonredundant list of the genes present in the 11 data sets. The FDR rate (Benjamini and Hochberg) was set to $\leq 0.1$.

\section{Signature enrichment visualisation}

See Additional file 2 for an outline of the process for integrating and visualizing analysis of gene signature and ontology enrichments.

The results from gene signature and gene ontology enrichment were used to create heatmap visualisations. For each meta-profile the top 100 most enriched signatures and 100 most enriched GO terms were used to construct a matrix of signatures against genes. This is a binary matrix with $1 \mathrm{~s}$ depicting an assigned signature/ GO annotation. Using Python a row-wise (gene correlation) and column-wise (signature correlation) phi coefficient was calculated. These were then hierarchical clustered using GENE-E [43] with complete linkage.

\section{Focus gene analysis}

See Additional file 3 for an outline of the focus gene approach.

Per data set the genes were ordered by their variance across the patient samples, and the top $80 \%$ were used to calculate Spearman's rank correlations per row using the Python scipy.stats package. The resultant $p$ value and correlation matrices were merged across the 11 data sets by taking the median values (across the sets in which the gene was contained), giving a final matrix of length 20,121. For a given focus gene the median rho and $p$ values were reported along with a breakdown of the correlations and relative expression levels across the data sets (Additional file 4). For select focus genes a correlated gene set was created by taking all genes with a $p>0.45$ present in six or more data sets. These correlated gene sets were then used for signature enrichment analysis and visualisation.

\section{Survival analysis}

The Survival library for $\mathrm{R}$ was used to analyse rightcensored survival data. Overall survival was estimated using the Kaplan-Meier method, modelled with Cox Proportional Hazards technique. Survival analysis was restricted to data sets of cases treated with the currently standard immunochemotherapy regimen R-CHOP. 


\section{Results}

Shared meta-profiles for COO-unclassified and COO-classified DLBCL

Given the importance of the COO paradigm to both the biological and clinical assessment of DLBCL, we anchored our initial analysis on this classification. We previously developed a $\mathrm{COO}$ classifier implementation that allows the robust classification of multiple DLBCL data sets [32], which is currently in clinical usage in the context of a phase 3 clinical trial [44]. Applying this to the 11 largest publicly available DLBCL data sets (GSE10846 was split according to treatment into CHOP and R-CHOP components), encompassing 2030 cases [15-25], provided a resource for gene expression metaanalysis. To determine genes consistently linked to COO class we used both the consistency of differential expression between data sets as well as absolute level of differential expression to identify and rank genes associated with each class. We restricted the gene lists by applying a threshold of differential expression in 6 out of 11 data sets; we refer to these as meta-profiles. To explore the relationship of COO-unclassified DLBCL to each of the principal $\mathrm{COO}$ classes, we employed sequential pairwise comparisons (Additional file 1). From the initial comparison, we identified 127 genes associated with COO-unclassified DLBCL relative to both $\mathrm{ABC}$ - and GCB-DLBCL, while 209 genes were associated with both
COO classes relative to COO-unclassified DLBCL (Additional file 5; Fig. 1). The extent of overlap was highly significant $(p=1.32 \mathrm{E}-157$ and $p=2.09 \mathrm{E}-200$ for genes associated with COO-unclassified DLBCL or COO class, respectively). We subsequently refer to these sets of overlapping genes as COO-unclassified and COO-classified meta-profiles, respectively.

\section{COO-unclassified DLBCL is enriched for features of a polarized immune response}

To assess underlying biology in the COO-classified and COO-unclassified meta-profiles we developed an approach for integrated analysis of GO and gene signature enrichment (Additional file 2) which applies hierarchical clustering to reciprocally assess the relationships of enriched ontology and signature terms and associated genes contributing to enrichments (Additional file 6). The results are displayed as heatmaps of the hierarchically clustered correlations.

In the COO-classified meta-profile a striking representation of genes linked to cell proliferation resulted in multiple distinct clusters of enriched terms reflecting a wide range of processes associated with cell proliferation (Fig. 2a; Additional file 7). In addition to this, distinct enrichment of signatures of the B-cell lineage was evident. From the gene perspective this was reflected in one main branch associated with cell cycle and cell
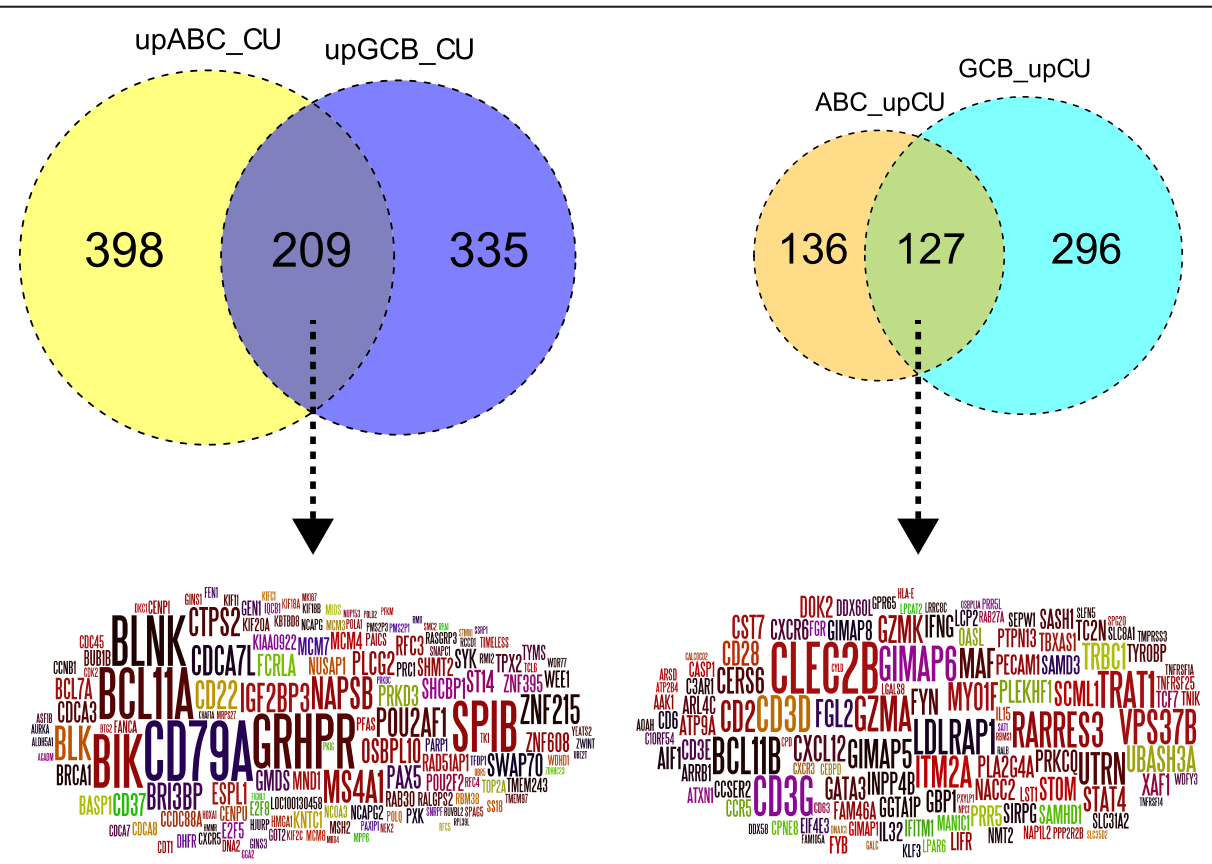

Fig. 1 Consistent gene expression differences separate COO-unclassified DLBCL from either principal COO class. The overlap of genes consistently associated with either COO-classified DLBCL (left Venn diagram and Wordle) or COO-unclassified DLBC (right Venn diagram and Wordle) are shown. Left: the Venn diagram shows genes up-regulated in ABC (yellow) or GCB (blue) relative to COO-unclassified. Right: the Venn diagram shows genes up-regulated in COO-unclassified relative to ABC-DLBCL (brown) or GCB-DLBCL (turquoise). For the Wordles, word size is given by differential expression (between contrasts) to the power of median-fold change 
A

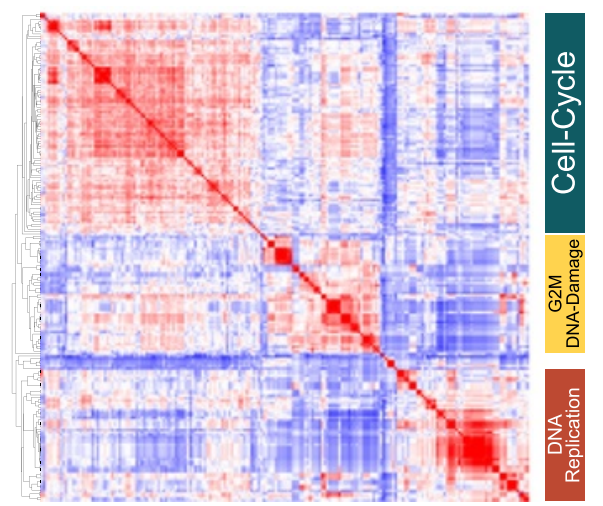

B

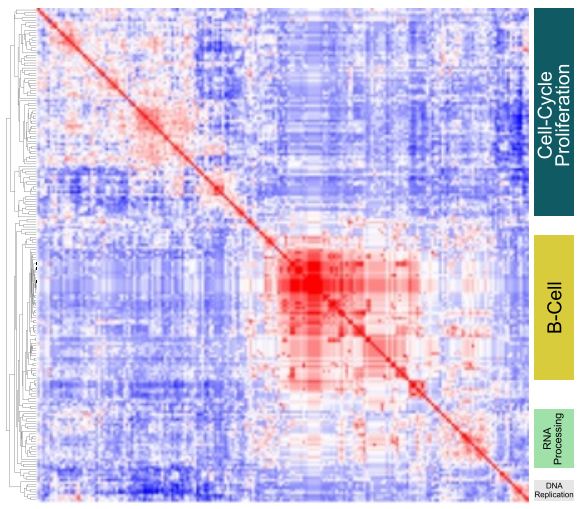

Fig. 2 Integrated gene signature and ontology enrichment analysis demonstrates association of the COO-classified meta-profile with cell proliferation and B-cell signatures. a The top gene signature and ontology terms enriched in the COO-classified meta-profile, clustered according to the correlation of signatures given their gene membership. $\mathbf{b}$ The corresponding clustering of genes contributing to signature and ontology term enrichments for the COO-classified meta-profile, clustered according to correlation of genes given their signature membership. To the right general categories corresponding to major correlation clusters are illustrated. Corresponding high resolution versions are available in Additional files 7 and 8

proliferation, and the second including two principal subclusters associated on the one hand with RNA binding and processing, and on the other with core B-cellassociated genes (Fig. 2b; Additional file 8).

In contrast the COO-unclassified meta-profile was linked to terms related to T-cell populations, T-cell receptor signalling and T-cell activation. While the second principal branch of ontology/signature terms was linked to additional more diverse immune response elements (Fig. 3a; Additional file 9). Hierarchical clustering from the gene perspective (Fig. 3b; Additional file 10) generated a principal branch related to $\mathrm{T}$ cells composed of a cluster of genes representing core elements of the T-cell state (CD2, CD3D, CD3E, CD3G, CD28 and TRBC1) and another cluster of genes with T-cell associations, including BCL11B, GZMA, GZMK, MAF and STAT4. The second principal branch of the hierarchical tree included genes derived from monocytes and other immune/host response signatures. This also included a subcluster comprising IFNG, and interferon responsive genes GBP1 and IFITM1, as well as the chemokine receptors CCR5, CXCR3 and CXCR6, which are linked to Th1 polarized T-cell populations $[45,46]$. We therefore conclude that COO-unclassified DLBCL is generally distinguished from COO-classified DLBCL by a predominant T-cell immune response with skewing toward IFNG gene
A

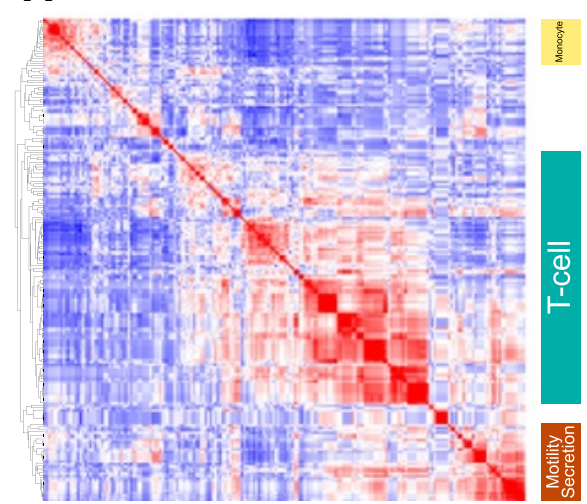

B

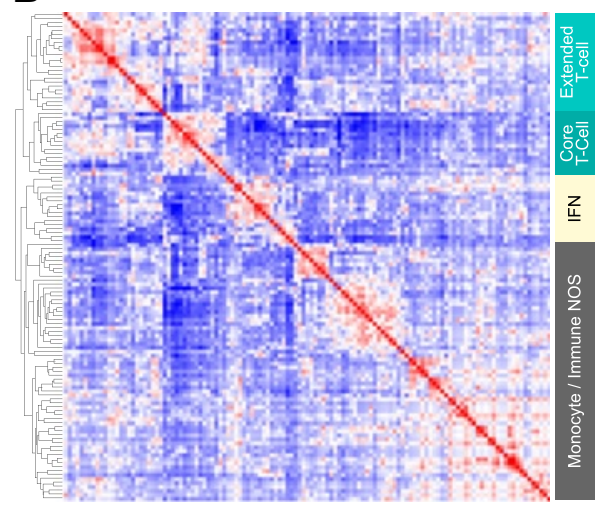

Fig. 3 Integrated gene signature and ontology enrichment analysis demonstrates association of the COO-unclassified meta-profile with polarized immune response. a The top gene signature and ontology terms enriched in the COO-unclassified meta-profile, clustered according to the correlation of signatures given their gene membership. $\mathbf{b}$ The corresponding clustering of genes contributing to signature and ontology term enrichments for the COO-unclassified meta-profile, clustered according to correlation of genes given their signature membership. To the right general terms corresponding to major correlation clusters are illustrated (NOS not otherwise specified). Corresponding high resolution versions are available in Additional files 9 and 10 
expression. Furthermore the paucity of both proliferation and B-cell gene expression is indicative of a relatively low representation of neoplastic B cells.

\section{A cytotoxic and interferon polarized immune response as} an independent molecular feature of DLBCL

We next addressed to what extent the identified polarized pattern of immune response was selective for COO-unclassified DLBCL or whether equivalently intense expression of polarized immune response genes might be detectable amongst some DLBCL cases that could be assigned to a principal COO class. As noted above, the COO-unclassified meta-profile separated on hierarchical clustering from the gene perspective into two branches, one of which was more strongly linked to core T-cell and cytotoxic genes (Fig. 4). To examine the relative ranking of genes belonging to these two hierarchical clustering branches within the COOunclassified meta-profile we superimposed the cluster membership onto scatter plots of differential expression ranking. We first ranked and then plotted genes belonging to the meta-profile by median fold differential expression in the comparison of COO-unclassified with ABC- or GCB-DLBCL. This demonstrated a significant overall correlation in the differential expression of COO-unclassified meta-profile genes relative to either principal COO class. Furthermore, genes belonging to the "T-cell cluster" (cluster 1) were significantly skewed toward most consistent association with COO-unclassified DLBCL (Additional file 11). To address whether the consistency of differential detection between data sets would alter this conclusion we ranked genes by a measure derived from both the number of data sets (consistency of differential expression) in which a gene was differentially expressed and the normalised median fold differential expression (Additional file 12). This again showed a significant overall correlation and a skewing of the T-cell cluster toward most consistent association with COOunclassified DLBCL $(p=6.57 \mathrm{E}-06$, hypergeometric test; Fig. 4). However, using either approach IFNG was identified as amongst the cluster 2 genes most consistently linked to COO-unclassified DLBCL.

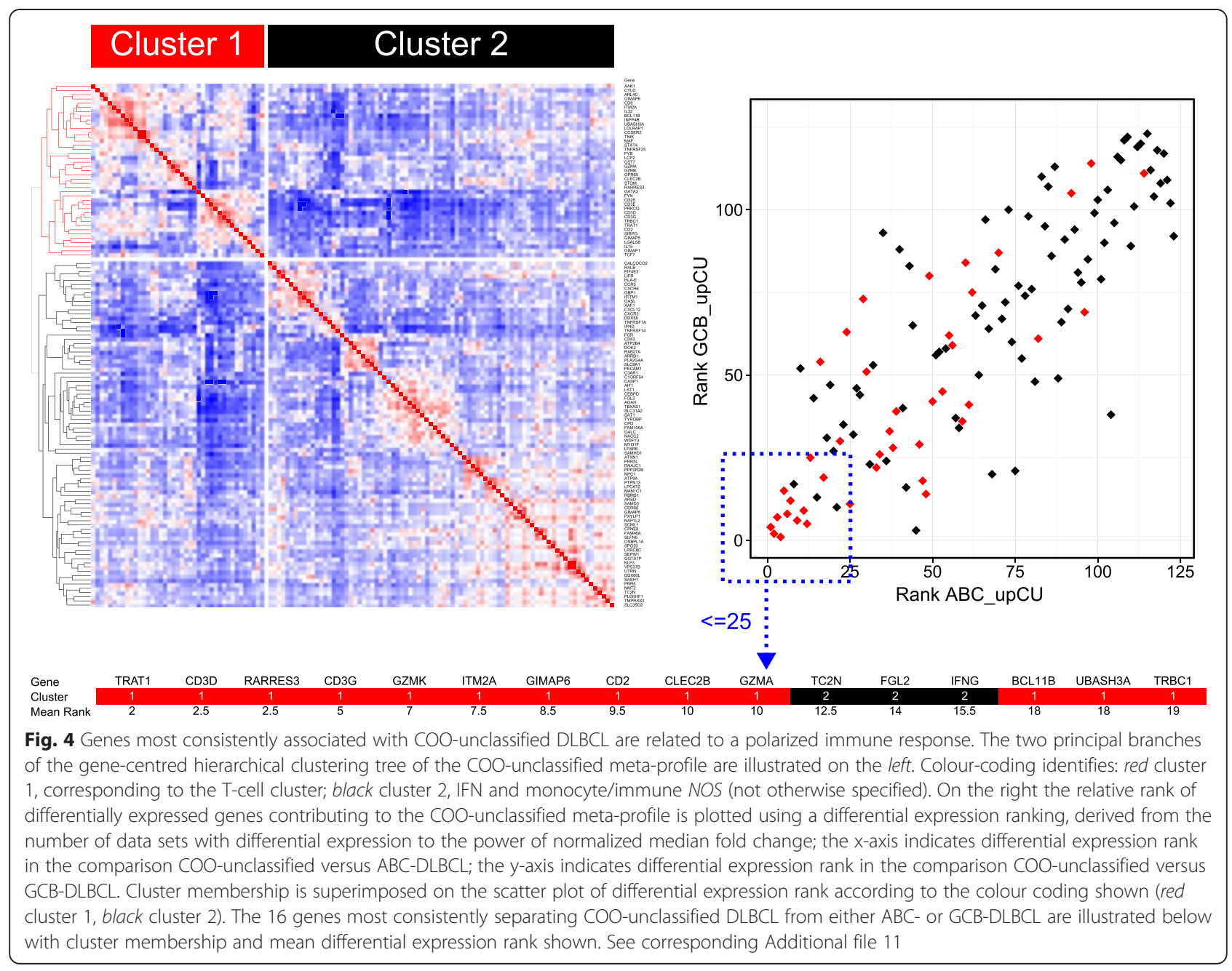


To examine the contribution of polarized immune response genes associated with COO-unclassified DLBCL across all data sets on a case-by-case basis we developed a linear additive classifier. For this we employed the 16 genes most strongly linked to COO-unclassified DLBCL derived from analysis using both the consistency/data set number and median fold differential expression. Given the contribution of core T-cell elements, cytotoxic genes and IFNG, we consider this to represent an integrated assessment of a polarized immune response. We ranked all cases in each data set by this linear score and plotted the incidence of cases classified as $\mathrm{ABC}, \mathrm{GCB}$ and unclassified on this ranking. Overall, individual COO-unclassified DLBCL cases showed a stronger association with the polarized immune response score relative to either $\mathrm{ABC}$ - or GCB-DLBCL (Fig. 5a; Additional file 13). This was particularly evident in the larger data sets GSE31312, GSE22470 and GSE10846. However, ABC- and GCBDLBCL cases with high levels of expression of the polarized immune response score were present in all data sets.
To assess whether the 16-gene score also reflected the expression of other genes associated with the immune response in COO-unclassified DLBCL we added further components of the meta-profile. Expression of these genes followed the overall pattern of expression of the 16-gene score across all DLBCL data sets (Fig. 5b; Additional file 13). Thus, the 16-gene score provides a tool with which to identify the overall pattern of this polarized immune response in DLBCL.

Since some COO-unclassified DLBCL cases in all data sets showed low polarized immune response scores, we examined the pattern of T-cell gene expression further by hierarchical clustering within each COO class. This demonstrated, particularly in the larger data sets such as GSE31312 and GSE22470, that COO-unclassified DLBCL could be segregated into principal groups with a subset of cases characterized both by weak expression of COO-classifier genes and weak expression of polarized immune response genes (Fig. 6; Additional file 14). Within the ABC- and GCB-DLBCL subsets there was a common concordance between expression of core T-cell genes and components of the polarized immune


Fig. 5 The polarized immune response is a dominant feature across DLBCL, independent of COO class. a The incidences of individual cases across all data sets (note GSE10846 is subdivided into CHOP and R-CHOP treated components) ranked according to polarized immune response score. The top and bottom 25 cases for each data set are illustrated with colour coding for COO class shown in the top bar (yellow ABC, blue GCB, green unclassified), class confidence assigned during classification shown in the middle bar (blue low confidence to red high confidence), and polarized immune response score shown in the bottom bar (blue low polarized immune response score to red high polarized immune response score). $\mathbf{b}$ Complete results for data sets GSE10846 R-CHOP and GSE31312, showing all cases ranked by polarized immune response score. Each heatmap displays class assignment, classification confidence and polarized immune response score summary as in (a) followed by COO-classifier gene expression (yellow and blue bars), the 16 genes of the polarized immune response score (green bar), and the extended set of COO-unclassified meta-profile genes (black bar). A corresponding high-resolution figure comprising equivalent representation for all data sets is provided in Additional file 13 


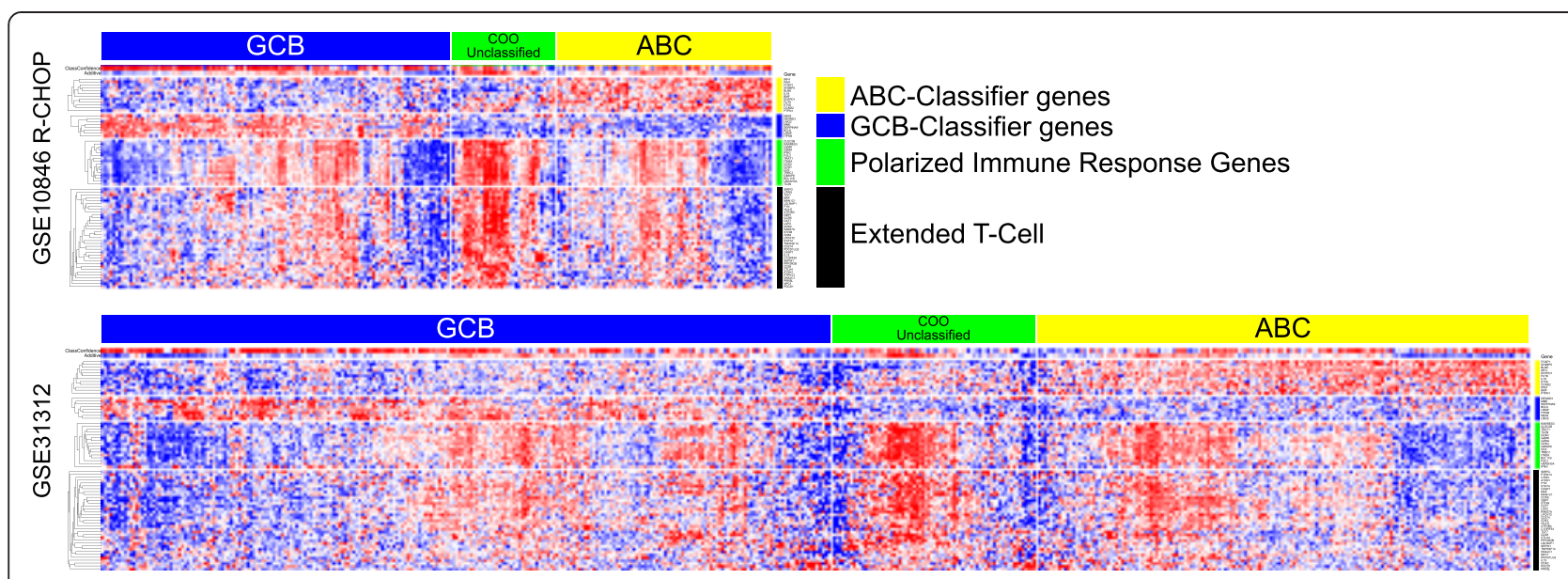

Fig. 6 The polarized immune response subdivides COO-unclassified DLBCL and identifies subsets of cases within ABC- and GCB-DLBCL classes. Heatmaps illustrate data for GSE10846 R-CHOP and GSE31312 hierarchically clustered according to all genes shown, and constrained by COO class assignment. Assigned COO class is shown above each heat map by the blue (GCB), green (COO-unclassified) and yellow (ABC) bars. To the right is shown the corresponding general category of genes: yellow ABC-classifier genes, blue GCB-classifier genes, green polarized immune response score genes, and black extended COO-unclassified meta-profile. A corresponding high-resolution figure comprising equivalent representation for all data sets is provided in Additional file 14

response. Only a few cases, particularly in the GCBDLBCL subset, could be identified in which core T-cell genes were co-expressed in the absence of other elements of the polarized response. These cases were, however, too few to allow meaningful analysis (data not shown). Thus, across all DLBCL data sets the expression of core T-cell genes is paralleled by the expression of genes linked to functional polarization irrespective of COO class.

Polarised immune response and COO-unclassified DLBCL do not overlap significantly with signatures of primary mediastinal B-cell lymphoma

COO-unclassified DLBCL cases lacking both polarized immune response and $\mathrm{COO}$-classifier gene expression are distinct from the subset of cases in which the extent of the polarized immune response obscures the characterization of the neoplastic B-cell population. At least two principal explanations could be considered for this subgroup: on the one hand these might include cases in which gene expression was technically challenging with poor representation of tumour cell RNA; alternatively, they might include a subset of large B-cell lymphoma which fails to express $\mathrm{COO}$-classifier genes at significant levels. Primary mediastinal B-cell lymphoma (PMBL) is a biologically distinct subgroup of large B-cell lymphoma, more common in women, with a mediastinal localization, distinct molecular genetics and possible derivation from a thymic B-cell population [47]. This lymphoma class can be associated with a pattern of gene expression distinct from either GCB- or ABC-DLBCL. While many PMBL cases would be excluded on the basis of diagnosis from conventional DLBCL gene expression data sets, it was possible that some PMBL cases might contribute to the COO-unclassified DLBCL cases, in particular those lacking a polarized immune response signature. To address this we used the 23-gene PMBL signature described by Rosenwald et al. [40], and first tested for enrichment within the COO-classified and COO-unclassified meta-profiles, but this showed no evidence of significant enrichment, nor was a signature separating PMBL from Hodgkin lymphoma enriched (Additional file 6). We next used the 23-gene PMBL signature in place of the extended immune response gene list to reanalyse the DLBCL data sets by hierarchical clustering (Additional file 15). We found no evidence of distinct clusters of cases identifiable with the 23-gene PMBL signature amongst COO-unclassified DLBCL, although a few elements of the 23-gene signature, most notably PDCD1LG2, CD274 and BATF3, do correlate with the polarized immune response. In contrast, in several data sets small clusters of cases were identifiable with coordinated high expression of the 23 genes of the PMBL signature, but such cases were classifiable as GCB-DLBCL, suggesting a greater overlap of PMBL signature gene expression amongst cases otherwise classifiable as GCB-DLBCL rather than ABC-DLCBL or COO-unclassified DLBCL. Thus, we found no gene expression-based evidence for a significant contribution of PMBL-like gene expression patterns amongst COOunclassified DLBCL in the data sets analysed. Inclusion of PMBL-like cases does not have a major impact on the detection of the polarized immune response signature, nor provide an explanation for the subset of COOunclassified DLBCL that lacks both COO-classifier and polarized immune response gene expression. 


\section{A polarized immune response is associated with improved outcome in DLBCL}

Across several cancer types the extent of tumour infiltrating lymphocytes, and their polarization toward cytotoxic $\mathrm{T} /$ natural killer (NK) cell gene expression linked to an IFN $\gamma$-STAT1-IRF1 signalling axis has been identified as a feature associated with good prognosis [4]. We therefore asked whether the expression of the polarized immune response signature, alone or taken in conjunction with $\mathrm{COO}$ class, was associated with differences in overall survival. Currently DLBCL is treated with an immunochemotherapy regimen, R-CHOP, which combines the anti-CD20 therapeutic monoclonal antibody rituximab with cyclophosphamide, hydroxydaunorubicin, vincristine (Oncovin), and prednisolone. Based on the success of the R-CHOP regimen, current treatment and future therapeutic trials in DLBCL will be based on immunochemotherapeutic approaches encompassing rituximab or related therapeutic antibodies. Therefore, only those data sets (GSE10846, GSE31312 and GSE32918) encompassing R-CHOP-treated cases associated with appropriate survival data were considered. This analysis demonstrated a consistent trend toward a reduced hazard ratio of death with increasing polarized immune response score across all three R-CHOP-treated DLBCL data sets. This reached statistical significance when considered independently of $\mathrm{COO}$ class in data sets GSE32918 and GSE31312, the latter representing the largest data set of R-CHOP-treated DLBCL [23]. However, in these two data sets the polarized immune response score was also significantly associated with lower age. When considered according to COO classification a consistent trend toward better outcome with high polarized immune response score was observed across all three categories. This trend was most pronounced for GCB-DLBCL, and reached statistical significance for improved outcome associated with high polarized immune response score in the largest data set GSE31312 (Additional file 16; Fig. 7). We conclude, therefore, that the presence of a polarized and IFN $\gamma$-associated immune response shows an association with good outcome which is modified by consideration of $\mathrm{COO}$ class, such that in the context of current R-CHOP therapy a polarized immune response is most consistently linked to improved outcome in patients with GCB-DLBCL.

\section{Polarization along an IFNY-STAT1-IRF1 axis is a defining feature of the DLBCL immune response}

While the above analysis pointed to a common convergence onto a cytotoxic and IFN $\gamma$-polarized immune response in DLBCL, not all components of the IFN $\gamma$ STAT1-IRF1 axis were sufficiently differentially expressed between COO-classified and COO-unclassified DLBCL to be identified by this approach. In order to explore the
DLBCL-associated immune response in a fashion which was not constrained by the COO paradigm we reanalyzed the DLBCL data sets, assessing the consistency and degree of correlated gene expression across all data sets relative to a selected "focus gene" (Fig. 8a; Additional files 3 and 4). We followed this by applying the integrated signature and GO enrichment analysis (Additional file 17).

As focus genes we selected two components of the 16gene polarized immune response signature, TRAT1 and $F G L 2$, to reflect origin from the two branches of the COO-unclassified meta-profile (Fig. 8b; Additional files 18 and 19). TRAT1 was selected as the most highly correlated gene from cluster 1 (Fig. 4), while FGL2 was selected as the second most highly correlated gene in cluster 2, and of more established immunologic interest than TC2N and less overt connection to immune response polarization than IFNG, the other two genes derived from cluster 2 that contribute to the 16-gene polarized immune response classifier.

Genes correlating with TRAT1 could be assigned to clusters of signatures and GO terms related to T-cell state, and T-cell signal transduction, cell motility and interferon response. For FGL2 as the focus gene a similar pattern emerged, including an expanded cluster of signature enrichments related to interferon responses, including some derived from models of viral infection, and an additional association with monocyte/macrophage-derived signatures.

To examine the strength of correlation with IFNresponsive genes we ranked genes by median correlation, plotted rank against median gene correlation for each focus gene context and assessed the distribution of selected IFN signature genes (derived from the previous analysis) on the resulting correlation curves. We applied this approach using TRAT1 and FGL2 as focus genes, but observed similar results with all 16 genes of the polarised immune response classifier (Fig. 9; Additional files 20). In either context IFN pathway genes were consistently present within the leading edge of most correlated genes, including IFNG, STAT1, IRF1, GBP1, GBP5 and IDO1. These genes were also consistently present within the leading edge when considering a more generic T/NK cell-associated gene, $C D 2$, as focus gene. Components of the IFN $\gamma$-STAT1-IRF1 axis therefore emerge as a consistent and dominant feature of the DLBCL immune environment linked to expression of a wider complement of IFN-responsive genes.

\section{IFNY-STAT1-IRF1 axis and adaptive immune regulatory pathways in DLBCL}

In the model of adaptive immune resistance IFN $\gamma$-driven expression of PD1 ligands CD274 and PDCD1LG2 on tumour cells and the microenvironment limits local T-cell responses $[5,8]$. We reasoned that the hierarchy of gene 
Total Data
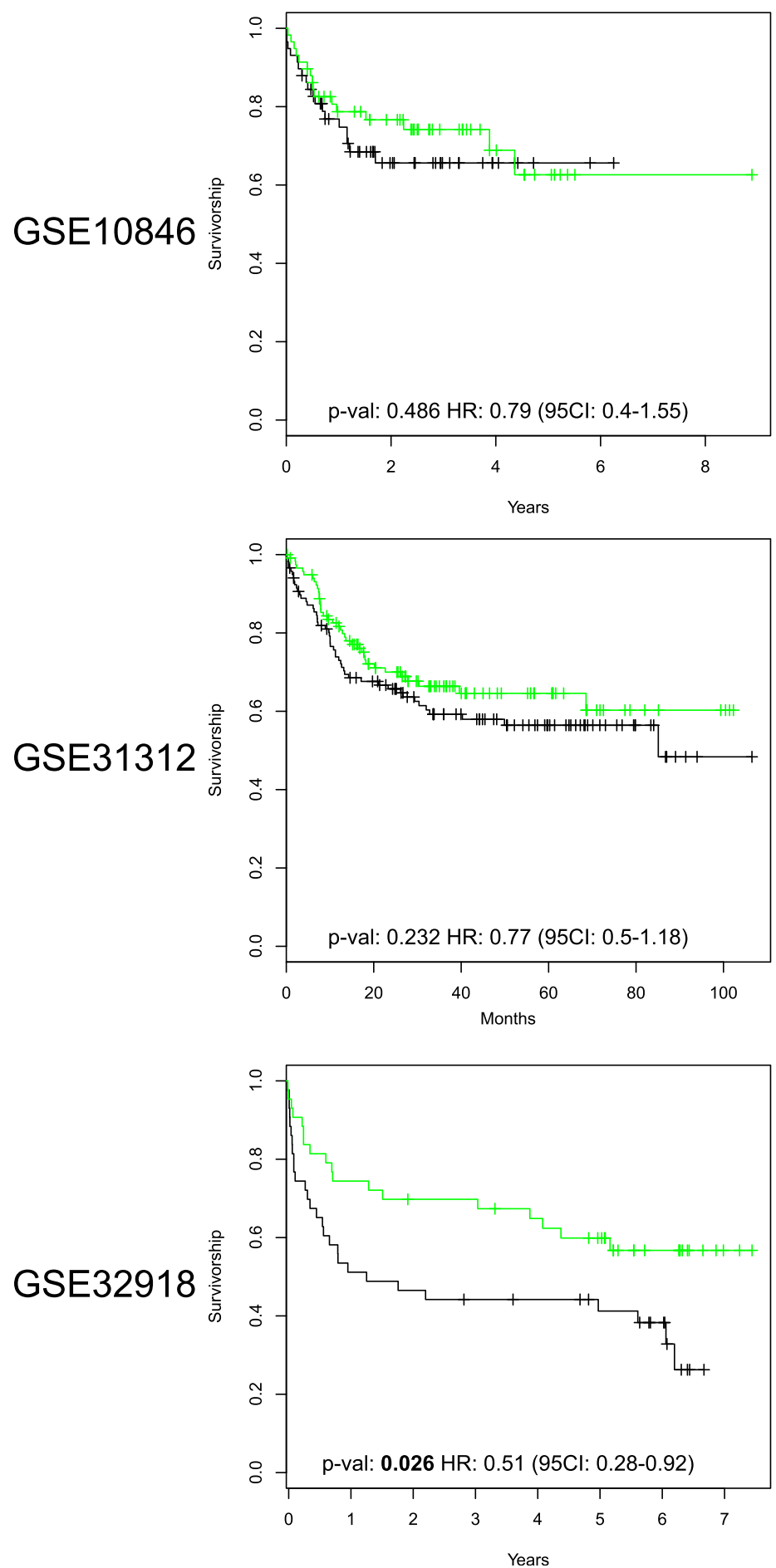


Fig. 7 A high polarized immune response score is associated with improved outcome in R-CHOP-treated GCB-DLBCL. The figure illustrates KaplanMeier plots of overall survival derived from R-CHOP-treated DLBCL cases from data sets GSE10846, GSE31312 and GSE32918. Illustrated is the overall survival for the top and bottom $25 \%$ of cases divided by polarized immune response score. The left graphs illustrate results independent of COO class and the right graphs results for cases assigned to the GCB-DLBCL class. Cl confidence interval, HR hazards ratio 


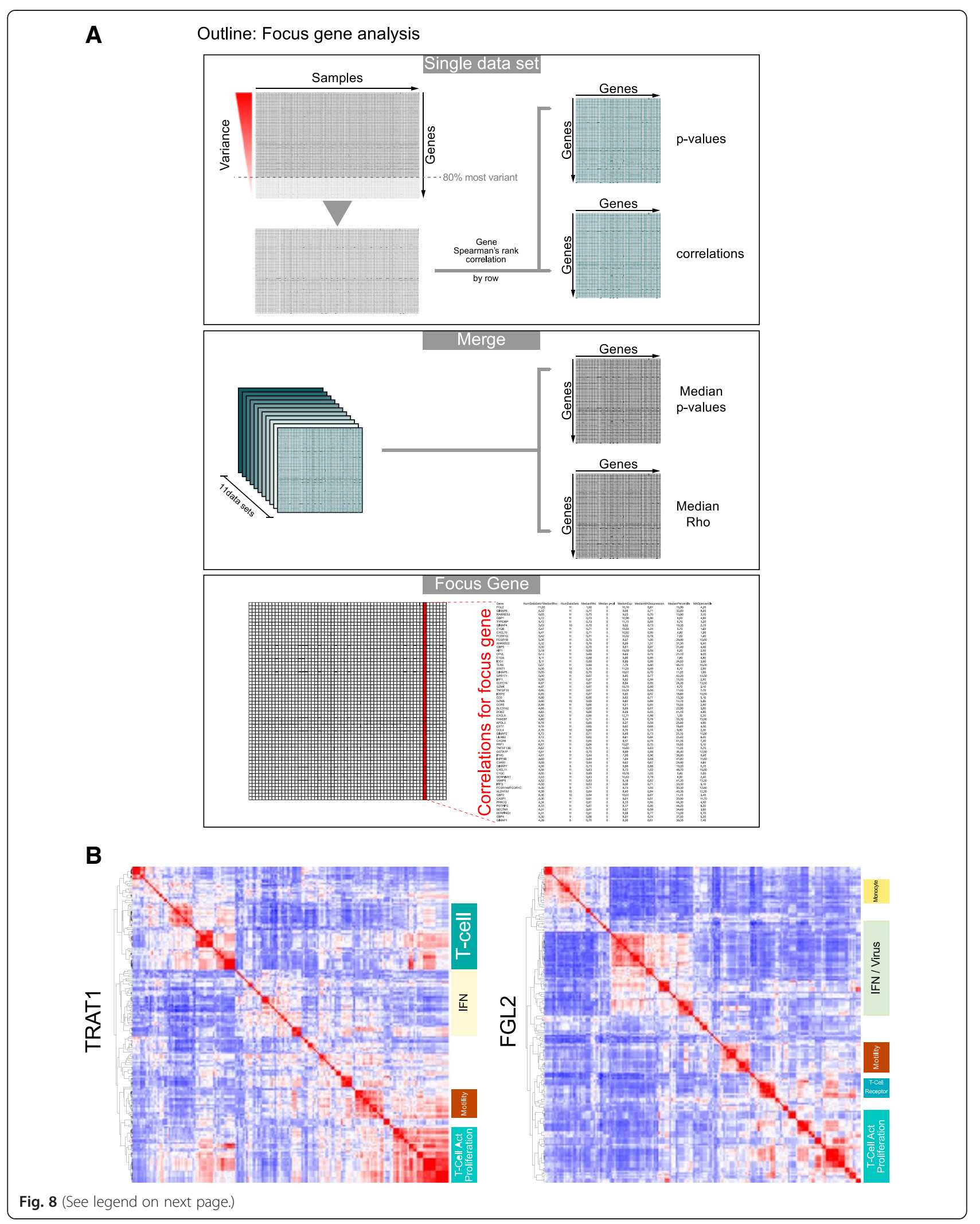


(See figure on previous page.)

Fig. 8 A focus gene analysis independent of $\mathrm{COO}$ class verifies the dominant polarized immune response in DLBCL. a An outline of the focus gene analysis (high resolution version in Additional file 3). Upper panel: the approach within each data set, with initial selection of the 80 \% most variable genes, and subsequent generation of linked matrices of gene correlation values and associated $p$ values. Middle panel: the merging of all data sets (11 data sets; data set GSE10846 subdivided by treatment type) is shown to give matrices of median correlations and $p$ values. Lower panel: the selection of an individual focus gene for downstream analysis. $\mathbf{b}$ Results of integrated gene signature and ontology analysis for two focus genes (left panel TRAT1) and (right panel FGL2) displaying the clustering of enriched signature and GO terms. General terms corresponding to major correlation clusters are illustrated to the right of each heatmap. Corresponding high resolution versions are available in Additional files 18 and 19, which also include the corresponding heatmaps clustered from the gene perspective

expression correlations would allow a ranking of immune checkpoint/modulatory gene expression linked to the IFN $\gamma$-STAT1-IRF1 polarized response in DLBCL. In this pathway STAT1 and IRF1 encode the transcriptional regulators; we therefore selected these along with $C D 2$ as a generic representative of the T/NK cell response for analysis (Fig. 10; Additional file 4). When considering immune modulatory/checkpoint genes a consistent cluster of three genes, LAG3, IDO1 and FGL2, emerged as most highly ranked and amongst the leading edge in all three focus gene contexts. In contrast, CD274 and PDCD1LG2 showed significantly weaker correlations with each focus gene, but nonetheless remained well correlated in comparison with all genes tested (rank $<1000$ out of 20,121 tested). To further confirm this pattern we extended the analysis to all 16 genes of the polarized immune response classifier, and observed similar patterns of gene correlation ranking (Additional file 21). Since the relative contribution of immune modulatory/checkpoint gene expression in tumour cells themselves relative to the wider microenvironment cannot be determined from these analyses, we conclude that, in addition to CD274 and PDCD1LG2, a wider complement of immune modulators provides a potentially high degree of redundancy in adaptive immune resistance in DLBCL. Amongst these components IDO1, FGL2 and LAG3 are particularly strongly correlated with IFN $\gamma$ polarized immune responses.

\section{Discussion}

The common convergence of cancer immune responses onto patterns of cytotoxic and IFN $\gamma$-dominated pathways has been summarised in the concept of an "immune constant of rejection" [4, 7]. In parallel the model of adaptive immune resistance argues for the control of such immune responses via local feedback driven through IFN-mediated upregulation of immune checkpoints $[5,8]$. Our analysis here provides extensive empirical evidence across currently available large DLBCL data sets that this combination of IFN $\gamma$ polarisation and induction of adaptive immune resistance mechanisms is a feature of the immune response to DLBCL. Unbiased analysis of gene expression correlations moreover suggests a hierarchy of IFN-associated immune modulatory gene expression with LAG3, IDO1 and FGL2 as key elements in this context. Thus, adaptive immune resistance is likely to provide an important component of immune evasion in DLBCL.

Other mechanisms of immune evasion have been previously identified as playing an important role in the pathogenesis of DLBCL, including mutation and deletion of $B 2 M$ and $C D 58$, and amplification of genomic regions encompassing genes encoding PD1 ligands [48, 49]. Furthermore previous studies have demonstrated the presence of PD1 expression on infiltrating T-cell populations and PD-L1(CD274) on tumour cells and in the microenvironment of DLBCL and related neoplasms [50, 51]. In the context of gene expression profiling, morphologically defined T-cell and histiocyte-rich large B-cell lymphoma, which represents a relatively rare subcategory, has been characterized by evidence of an IFNassociated immune response, linked on the one hand with over-expression of PD1 (PDCD1) on infiltrating $\mathrm{T}$ cells when compared with classical Hodgkin lymphoma [52], or the expression of IDO1 when compared with nodular lymphocyte predominant Hodgkin lymphoma, another relatively rare lymphoma subtype [53]. Indeed, expression of IDO1 has been defined as a feature associated with poor outcome in generic DLBCL in one patient series [54]. Thus, the involvement of several pathways of immune modulation in large B-cell lymphomas is supported by prior studies.

Using the 16-gene polarized immune response score we have ranked DLBCL cases across multiple data sets, and demonstrate that a substantial fraction of cases regardless of $\mathrm{COO}$ class are linked to a polarized immune response. In the context of the $\mathrm{COO}$ classification, the dominance of this immune response at the expense of proliferating $\mathrm{B}$ cells provides the most common explanation for DLBCL cases that are "unclassifiable" as originally suggested by Monti et al. [15]. Equally important is the identification of a distinct group of DLBCL characterized by an absence of host response elements, which is consistent with "immunological ignorance", a feature which in other cancers is associated with poor response to immune checkpoint blockade [12]. These cases are also consistent with a model of host tissue "effacement" proposed by Scott and Gascoyne [49] as distinguishing subsets of aggressive lymphomas. Immune 





(See figure on previous page.)

Fig. 9 IFN-responsive genes and the IFNY-STAT1-IRF1 axis are amongst the leading edge of highly correlated DLBCL immune response genes. Correlation curves were generated from the focus gene analysis by ranking genes according to median correlation, and then plotting the gene correlation rank (x-axis) against the corresponding median gene correlation (y-axis, median Rho). This illustrates both the relative strength of correlations for each focus gene and identifies a leading edge of genes with most significant correlations. The position of a set of IFN-associated genes was plotted for each focus gene context as indicated in the figure. Note only the top 2000 of 20,121 genes tested are illustrated. See corresponding Additional file 20

evasion in DLBCLs can be associated with loss of MHC class I expression consequent on mutation and/or deletion of $B 2 M$, which may be further accompanied by inactivation of CD58 [48], and a prediction might be that such cases would be enriched in the subset characterized by apparent immunological ignorance. However, analogous lesions affecting $B 2 M$ were recently identified as recurrent events positively associated with cytotoxic gene signatures in solid tumours [9]. This suggests a model in which adaptive immune resistance mechanisms may be followed by somatic genetic alterations that further enhance tumour immune escape. Whether a similar positive association between cytotoxic response and $B 2 M$ or $C D 58$ mutation status exists in DLBCL is, to our knowledge, not established.

Across several cancer types the intensity of tumour infiltrating lymphocytes and their functional polarization has proved to be of prognostic significance in the absence of specific immune checkpoint blockade [4, 55-57]. Our analysis indicates that a trend toward an improved outcome in association with a more intense polarized immune response is generally maintained in the context of DLBCL treated with the current immunochemotherapy regimen, $\mathrm{R}-\mathrm{CHOP}$. However, this benefit is not equivalent across all DLBCL when considered in relation to $\mathrm{COO}$ class, and is most pronounced for GCB-DLBCL. Indeed, in the largest available data set of R-CHOP-treated DLBCL, GSE31312 [23], a substantial group of patients with both a GCB-DLBCL classification and a high polarized immune response score appeared curable with current therapy. As a statistically significant association is not consistently observed across all three data sets of DLBCL treated with R-CHOP, and there is a potentially confounding association with young age, the overall prognostic value of this association remains uncertain in the context of current therapy. Additional features of the host response, which did not emerge as principal discriminants between COO-classified versus COO-unclassified DLBCL, such as contributions from macrophage/monocyte lineage cells, may add value to immune response classifiers. These will need to be considered alongside the polarized immune response signature in future work. Nonetheless, the analysis presented here demonstrates a graded pattern of immune response in DLBCL, with one extreme characterized by minimal cytotoxic immune response signature and tendency to poor outcome, and another extreme characterized by intense polarized immune response and a tendency toward better outcome which is modified by $\mathrm{COO}$ class. In other settings the pattern of pre-existing immune response prior to immune checkpoint therapy has proved to be of predictive value $[11,12,58,59]$. Based on this evidence, it is the subset of DLBCL cases with preexisting polarized immune response which is most likely to benefit from immune checkpoint/modulatory therapy, and is readily identifiable in a quantitative fashion from gene expression data.

Immune checkpoint inhibitors are already under evaluation in the context of large cell lymphomas [60, 61]. Recent clinical trials with PD1 pathway blockade have shown substantial promise in Hodgkin lymphoma [14], as in other tumour types $[11,12,62]$. Combining immune checkpoint inhibitors may hold particular promise, and both LAG3 and IDO1 are therapeutic targets with novel agents in current clinical evaluation. Our analyses support these as high priority candidates for therapeutic evaluation in DLBCL alongside PD1 blockade. In addition to direct interventions specifically targeting immune checkpoints, signalling pathways that mediate survival of neoplastic B cells, and are the targets of novel therapeutic agents in lymphoma, overlap with pathways controlling T-cell responses. Such agents have the potential to de-repress cytotoxic T-cell populations and promote anti-tumour immunity [63]. Thus, companion biomarkers evaluating the potential association between pre-existing immune response at diagnosis and treatment response should arguably also be included in the setting of lymphoma clinical trials where agents targeting lymphocyte signalling pathways are being evaluated.

A notable element of the DLBCL immune response is the consistent association with FGL2 expression. This encodes fibrinogen-like 2 prothrombinase, a protein that has dual roles as a pro-coagulant and immune modulator. FGL2 has been shown to act as an immune responsive coagulant in settings such as foetal loss driven by Th1 polarized immune responses [64] and fulminant hepatitis [65]. Subsequently, FGL2 has been implicated as a repressor of $\mathrm{T}$-cell activation both in the ability of recombinant FGL2 to block graft rejection [66] and in the context of Fgl2 knockout mice developing autoimmune glomerulonephritis [67]. In several experimental models FGL2 has been associated with suppression of cytotoxic and Th1-polarized immune responses [67-69]. FGL2 effects in DLBCL could relate to both pro-coagulant and immune modulatory functions. In DLBCL FGL2 



Fig. 10 (See legend on next page.) 
(See figure on previous page.)

Fig. 10 Immune-modulatory and checkpoint gene expression is strongly correlated with elements of the IFNY-STAT1-IRF1 axis in DLBCL. IRF1 and STAT1 along with CD2 were analysed as focus genes, and resultant correlation curves are illustrated. Genes were plotted according to correlation rank (x-axis) against median gene correlation ( $y$-axis, median Rho). The position of immune checkpoint/modulatory genes on the resulting curves was plotted for each focus gene as indicated in the figure. Note only the top 2000 of 20,121 genes tested are illustrated. See corresponding Additional file 21

expression correlates with multiple elements of the IFN $\gamma$ STAT1-IRF1 axis; supporting direct regulation, FGL2 expression has previously been shown to be responsive to IFNy in T cells [70, 71], and was shown to act downstream of IRF1 in Th1-driven foetal loss [64]. Thus, the relationships in DLBCL suggest that FGL2 may provide an additional element of negative feedback and adaptive immune resistance, which is potentially suitable for therapeutic targeting [72, 73].

We note that some DLBCL cases with a prominent immune response may be associated with Epstein-Barr virus (EBV) infection and oncogenic drive. In the metaanalysis approach taken here the contribution of EBV cannot be systematically assessed from available data since EBV status is incompletely annotated, and not necessarily assessed using both immunohistochemistry for EBV LMP1 and RNA-FISH for EBERs. Immune surveillance is known to contribute to the control of EBVmediated tumours [74], and the presence of high EBV loads can contribute to the establishment of an exhausted cytotoxic response [75]. Indeed, there are significant overlaps between the gene expression profiles of the immune response in EBV-associated large cell lymphomas occurring in the post-transplant setting [76] and the polarized IFN $\gamma$-associated gene expression that is evident from our DLBCL meta-analysis. However, while the frequency of EBV infection in generically diagnosed DLBCL has been established at close to $10 \%$ [77], significant expression of genes linked to the polarized immune response is more frequent across DLBCL data sets. An overlap of gene expression profiles between the immune response targeting EBV-driven and EBV-independent lymphomas is consistent with the model of convergent patterns of "immune rejection" across diverse immune contexts [4, 7]. It is arguable that the principal predictive factor of response to immune checkpoint inhibition will be the presence of a pre-existing polarized immune response and the mechanisms controlling its chronic activation/exhaustion rather than the nature of the initial triggering antigens whether viral or cancer-associated.

\section{Conclusions}

The analysis presented here supports the central importance of convergent patterns of immune response linked to the IFN $\gamma$-STAT1-IRF1 axis, coupled to IFN-driven feedback pathways in DLBCL. This argues for the generalisable nature of these interconnected mechanisms, and implicates a hierarchy of immune modulators, known to promote the establishment of an immunosuppressive microenvironment [2], in the process of IFN $\gamma$-driven adaptive immune resistance.

\section{Additional files}

Additional file 1: Figure S1. Outline of meta-profile generation using COO classification. Upper panel: the data sets used. Please note one data set, GSE10846, is divided into two component parts reflecting underlying differences in treatment (CHOP versus $\mathrm{R}-\mathrm{CHOP}$ ), giving a total of 11 separate data set components. Illustrated are all cases for each data set, subdivided by $\mathrm{COO}$ classification established using the DAC classifier [32] and ranked by classification confidence, with classifier genes illustrated on the right (yellow bars ABC classifier genes and cases, blue bars GCB classifier genes and cases, green bars COO-unclassified cases). Middle panels: the pairwise comparisons of differentially expressed genes between three classes, and the integration of differentially expressed genes across data sets. Bottom panels: the resulting meta-profiles of differentially expressed genes for both components of the three possible pairwise comparisons are shown as Wordles for illustrative purposes (complete details provided in Additional file 5). (PDF 2024 kb)

Additional file 2: Figure S2. Outline of the process for integrating and visualizing analysis of gene signature and ontology enrichments. The flow diagram illustrates the process for integrating gene signature and ontology enrichments. The initial assessment of overlap between meta-profiles derived from the comparison of $A B C-D L B C L$ versus COO-unclassified (CU) DLBCL and GCB-DLBCL versus COO-unclassified (CU) DLBCL is shown at the top of the figure, followed by the parallel analysis of gene ontology (BiNGO) and hypergeometric testing of signature enrichments. Next a matrix is illustrated showing the occurrence of genes versus enriched signatures (green fill), followed by analysis of correlations (Phi coefficient) by column (signature/ontology terms) or by row (genes) and hierarchical clustering. (PDF $1658 \mathrm{~kb}$ )

Additional file 3: Figure S11. An outline of the focus gene approach, and a high resolution image to accompany Fig. 8a. Upper panel: the approach within each data set with initial selection of the $80 \%$ most variable genes, and subsequent generation of linked matrices of gene correlation values and associated $p$ values. Middle panel: merging of all data sets (11 data sets; data set GSE10846 subdivided by treatment type) to give gene by gene matrices of median correlations and $p$ values. Lower panel: the selection of an individual focus gene for downstream analysis. (PDF $1375 \mathrm{~kb}$ )

Additional file 4: Table S5. Lists of correlated genes for selected focus genes. (XLSX 16708 kb)

Additional file 5: Table S1. Lists of Meta-profile genes differentially expressed between DLBCL COO classes. (XLSX 2318 kb)

Additional file 6: Table S2. Lists of enriched gene signature and gene ontology terms for COO-classified and COO-unclassified meta-profiles. (XLSX $2848 \mathrm{~kb}$ )

Additional file 7: Figure S3. High resolution image corresponding to Fig. 2a. Integrated gene signature and ontology enrichments for COOclassified meta-profile clusters from signature and ontology term perspectives. The figure represents the hierarchical clustering of enriched gene signature and ontology terms related to the COO-classified meta-profile. Correlations are illustrated in heat maps on a blue (least) to red (most) scale as indicated at the top of the figure. Along the edges of the heatmap the signature terms are provided (and correspond to terms listed in Additional file 6). The FDRcorrected $p$ value for enrichment of the signature is illustrated as a bar on either side of the heatmap, along with an indication of the type of term 
(signature versus ontology) and the origin of the terms as indicated in the figure. (PDF $283 \mathrm{~kb}$ )

Additional file 8: Figure S4. High resolution image corresponding to Fig. 2b. Integrated gene signature and ontology enrichments for $\mathrm{COO}-$ classified meta-profile clustered from the gene perspective. The figure represents the hierarchical clustering of meta-profile genes contributing to signature and ontology term enrichments, and clustered according to the correlation of enriched signature/ontology term membership. Correlations are illustrated in the heatmap on a blue (least) to red (most) scale as indicated at the top of the figure. Along the edges of the heatmap official gene symbols are provided. (PDF $251 \mathrm{~kb}$ )

Additional file 9: Figure S5. High resolution image corresponding to Fig. 3a. Integrated gene signature and ontology enrichments for $\mathrm{COO}$ unclassified meta-profile clusters from signature and ontology term perspectives. The figure represents the hierarchical clustering of enriched gene signature and ontology terms related to the COO-unclassified meta-profile. Correlations are illustrated in heat maps on a blue (least) to red (most) scale as indicated at the top of the figure. Along the edges of the heatmap the signature terms are provided (and correspond to terms listed in Additional file 6). The FDR-corrected $p$ value for enrichment of the signature is illustrated as a bar on either side of the heatmap, along with an indication of the type of term (signature versus ontology) and the origin of the terms as indicated in the figure. (PDF $518 \mathrm{~kb}$ )

Additional file 10: Figure S6. High resolution image corresponding to Fig. 3b. Integrated gene signature and ontology enrichments for COOunclassified meta-profile clustered from the gene perspective. The figure represents the hierarchical clustering of meta-profile genes contributing to signature and ontology term enrichments, and clustered according to the correlation of enriched signature/ontology term membership. Correlations are illustrated in the heatmap on a blue (least) to red (most) scale as indicated at the top of the figure. Along the edges of the heatmap official gene symbols are provided. (PDF $126 \mathrm{~kb}$ )

Additional file 11: Figure S7. Relates to Fig. 4. Genes most consistently associated with COO-unclassified DLBCL are related to a polarized immune response. As in Fig. 4, the two principal branches of the gene-centred hierarchical clustering tree of the COO-unclassified meta-profile are illustrated on the left. Colour-coding above identifies: red cluster 1, corresponding to the T-cell cluster; black cluster 2, IFN and monocyte/ immune NOS (not otherwise specified). On the right the relative rank of differentially expressed genes contributing to the COO-unclassified metaprofile is plotted using the median normalized fold change for gene ranking; the $x$-axis indicates differential expression rank in the comparison COO-unclassified versus ABC-DLBCL; the $y$-axis indicates differential expression rank in the comparison COO-unclassified versus GCB-DLBCL. Cluster membership is superimposed on the scatter plot of differential expression rank according to the colour coding shown (red cluster 1, black cluster 2). The 18 genes most consistently separating COO-unclassified DLBCL from either ABC- or GCB-DLBCL are illustrated below with cluster membership and mean differential expression rank shown. (PDF $275 \mathrm{~kb}$ )

Additional file 12: Table S3. Ranked list of genes contributing to shared meta-profiles of COO-classified and COO-unclassified DLBCL. (XLSX $38 \mathrm{~kb}$ )

Additional file 13: Figure S8. Relates to Fig. 5. Ranking by the 16-gene polarized immune response score demonstrates common occurrence of a polarized T-cell response across DLBCL from all data sets. Shown are all $D L B C L$ data sets used with cases ranked by the 16-gene polarized immune response score. The data set number is shown above each heat map, followed by three bars: top bar COO class (yellow ABC, blue GCB, green unclassified); middle bar class confidence assigned during classification (blue low confidence to red high confidence); bottom bar polarized score (blue low polarized immune response score to red high polarized immune response score). These are followed by case-by-case gene expression values (illustrated as z scores) which are broken down into components identified by coloured bars on the right of each heatmap. The contributing genes are shown in the grey expanded box to the right of the figure with corresponding colour code: yellow bar ABC COO-classifier genes; blue bar GCB COO-classifier genes; green bar polarized immune response score; black bar extended COO-unclassified meta-profile and immune response genes. (PDF $1073 \mathrm{~kb}$ )

Additional file 14: Figure S9. Relates to Fig. 6. Clustering within COO classes demonstrates subdivision of unclassified DLBCL by polarized immune response score, and occurrence of immune response-rich cases in each principal COO class. Shown are all DLBCL data sets used, hierarchically clustered by all genes shown, and constrained by COO class. The data set number is shown above each heatmap, followed by three bars: top bar COO class (yellow ABC, blue GCB, green unclassified); middle bar class confidence assigned during classification (blue low confidence to red high confidence); bottom bar polarized score (blue low polarized immune response score to red high polarized immune response score). These are followed by case-by-case gene expression values (illustrated as z scores), which are broken down into components identified by coloured bars on the right of each heatmap. The contributing genes are shown in the grey expanded box to the right of the figure with corresponding color code: yellow bar ABC COO-classifier genes; blue bar GCB COO-classifier genes; green bar polarized immune response score; black bar extended COOunclassified meta-profile and immune response genes. (PDF $1168 \mathrm{~kb}$ )

Additional file 15: Figure S10. Clustering of $\mathrm{COO}$ classes and PMBL signature genes. Shown are all DLBCL data sets used, hierarchically clustered by all genes shown, including the 23-gene PMBL signature, and constrained by $\mathrm{COO}$ class. The data set number is shown above each heatmap, followed by three bars: top bar $\mathrm{COO}$ class (yellow ABC, blue GCB, green unclassified); middle bar class confidence assigned during classification (blue low confidence to red high confidence); bottom bar polarized score (blue low polarized immune response score to red high polarized immune response score). These are followed by case-bycase gene expression values (illustrated as z scores), which are broken down into components identified by coloured bars on the right of each heatmap. The contributing genes are shown in the grey expanded box to the right of the figure with corresponding color code: yellow bar ABC COO-classifier genes; blue bar GCB COOclassifier genes; green bar polarized immune response score; black bar PMBL 23-gene signature. (PDF 1002 kb)

Additional file 16: Table S4. Details for survival/outcome data related to the linear score. (XLSX $17 \mathrm{~kb}$ )

Additional file 17: Table S6. Signature and ontology enrichments for the leading edge of most correlated genes for each focus gene context. (XLSX $2731 \mathrm{~kb}$ )

Additional file 18: Figure S12. Relates to Fig. 8b. Integrated gene signature and ontology enrichments for TRAT1 focus gene analysis. Upper panel: the hierarchical clustering of enriched gene signature and GO terms related to genes correlating with TRAT1 in focus gene analysis. Correlations are illustrated in the heatmap on a blue (least) to red (most) scale as indicated at the top of the figure. Along the edges of the heatmap the signature terms are provided (and correspond to terms listed in Additional file 17). The FDR-corrected $p$ value for enrichment of the signature is illustrated as a bar on either side of the heatmap, along with an indication of the type of term (signature versus ontology) and the origin of the terms as indicated in the figure. Lower panel: the hierarchical clustering of genes correlating with TRAT1 in focus gene analysis. Genes shown are clustered according to the correlation of enriched signature/GO term membership. Correlations are illustrated in the heatmap on a blue (least) to red (most) scale as indicated at the top of the figure. Along the edges of the heatmap official gene symbols are provided. (PDF $1104 \mathrm{~kb}$ )

Additional file 19: Figure S13. Relates to Fig. 8b. Integrated gene signature and ontology enrichments for FGL2 focus gene analysis. Upper panel: the hierarchical clustering of enriched gene signature and $\mathrm{GO}$ terms related to genes correlating with FGL2 in focus gene analysis. Correlations are illustrated in the heatmap on a blue (least) to red (most) scale as indicated at the top of the figure. Along the edges of the heatmap the signature terms are provided (and correspond to terms listed in Additional file 17). The FDR-corrected $p$ value for enrichment of the signature is illustrated as a bar on either side of the heatmap, along with an indication of the type of term (signature versus ontology) and the origin of the terms as indicated in the figure. Lower panel: the hierarchical clustering of genes correlating with FGL2 in focus gene analysis. Genes shown are 
clustered according to the correlation of enriched signature/GO term membership. Correlations are illustrated in the heatmap on a blue (least) to red (most) scale as indicated at the top of the figure. Along the edges of the heatmap official gene symbols are provided. (PDF $1772 \mathrm{~kb}$ )

Additional file 20: Figure S14. Relates to Fig. 9. IFN-responsive genes and the IFNY-STAT1-IRF1 axis are amongst the leading edge of highly correlated DLBCL immune response genes. Correlation curves were generated from the focus gene analysis for all 16 genes used in the polarized immune response signature by ranking genes according to median correlation, and then plotting the gene correlation rank ( $x$-axis) against the corresponding median gene correlation (y-axis, median Rho). This illustrates both the relative strength of correlations for each focus gene and identifies a leading edge of genes with most significant correlations. The position of a set of IFN-associated genes was plotted for each focus gene context as indicated in the figure. Note only the top 2000 of 20,121 genes tested are illustrated. (PDF $145 \mathrm{~kb}$ )

Additional file 21: Figure S15. Relates to Fig. 10. Immune-modulatory and checkpoint gene expression is strongly correlated with elements of the polarized immune response signature. Correlation curves were generated from the focus gene analysis for all 16 genes used in the polarized immune response signature by ranking genes according to median correlation, and then plotting the gene correlation rank ( $x$-axis) against the corresponding median gene correlation ( $y$-axis, medianRHO). The position of immune checkpoint/modulatory genes on the resulting curves was plotted for each focus gene as indicated in the figure. Note only the top 2000 of 20,121 genes tested are illustrated. (PDF $140 \mathrm{~kb}$ )

\section{Abbreviations}

ABC: activated B cell; CHOP: cyclophosphamide, doxorubicin hydrochloride (hydroxydaunomycin),vincristine sulfate (Oncovin), prednisone; COO: cell of origin; DLBCL: diffuse large B-cell lymphoma; EBV: Epstein-Barr virus; FDR: false discovery rate; GCB: germinal centre B cell; GEO: Gene Expression Omnibus; GO: gene ontology; HGNC: HUGO Gene Nomenclature Committee; IFN: interferon; NK: natural killer; PMBL: primary mediastinal B-cell lymphoma; R-CHOP: rituximab-CHOP.

\section{Competing interests}

The authors declare that they have no competing interests.

\section{Authors' contributions}

MAC designed and performed analyses, analysed data and wrote the manuscript; DRW oversaw bioinformatics analyses and edited manuscript; RT designed and oversaw the study, analysed data and wrote the manuscript. All authors read and approved the final manuscript.

\section{Acknowledgements}

This work was supported by a Cancer Research UK senior clinical fellowship to RMT (C7845/A10066) and Cancer Research UK programme grant C7845/ A17723. We thank Dr Gina Doody, Dr Andrew Jack and Dr Darren Newton for critical review.

\section{Author details}

'Section of Experimental Haematology, Wellcome Trust Brenner Building, Leeds Institute of Cancer and Pathology, University of Leeds, Leeds LS9 7TF, UK. ${ }^{2}$ Bioinformatics Group, School of Molecular and Cellular Biology, University of Leeds, Leeds, UK.

\section{Received: 18 March 2015 Accepted: 17 August 2015 Published online: 11 September 2015}

\section{References}

1. Zitvogel L, Galluzzi L, Smyth MJ, Kroemer G. Mechanism of action of conventional and targeted anticancer therapies: reinstating immunosurveillance. Immunity. 2013;39:74-88. doi:10.1016/j.immuni.2013.06.014.

2. Vesely MD, Kershaw MH, Schreiber RD, Smyth MJ. Natural innate and adaptive immunity to cancer. Annu Rev Immunol. 2011;29:235-71. doi:10.1146/annurev-immunol-031210-101324.

3. Speiser DE, Utzschneider DT, Oberle SG, Munz C, Romero P, Zehn D. T cell differentiation in chronic infection and cancer: functional adaptation or exhaustion? Nat Rev Immunol. 2014;14:768-74. doi:10.1038/nri3740.
4. Galon J, Angell HK, Bedognetti D, Marincola FM. The continuum of cancer immunosurveillance: prognostic, predictive, and mechanistic signatures. Immunity. 2013;39:11-26. doi:10.1016/j.immuni.2013.07.008.

5. Pardoll DM. The blockade of immune checkpoints in cancer immunotherapy. Nat Rev Cancer. 2012;12:252-64. doi:10.1038/nrc3239.

6. Page DB, Postow MA, Callahan MK, Allison JP, Wolchok JD. Immune modulation in cancer with antibodies. Annu Rev Med. 2014;65:185-202. doi:10.1146/annurev-med-092012-112807.

7. Wang E, Worschech A, Marincola FM. The immunologic constant of rejection. Trends Immunol. 2008;29:256-62. doi:10.1016/j.it.2008.03.002.

8. Taube JM, Anders RA, Young GD, Xu H, Sharma R, McMiller TL, et al. Colocalization of inflammatory response with B7-h1 expression in human melanocytic lesions supports an adaptive resistance mechanism of immune escape. Sci Transl Med. 2012;4:127ra37. doi:10.1126/ scitranslmed.3003689.

9. Rooney MS, Shukla SA, Wu CJ, Getz G, Hacohen N. Molecular and genetic properties of tumors associated with local immune cytolytic activity. Cell. 2015;160:48-61. doi:10.1016/j.cell.2014.12.033.

10. Wolchok JD, Kluger H, Callahan MK, Postow MA, Rizvi NA, Lesokhin AM, et al. Nivolumab plus ipilimumab in advanced melanoma. N Engl J Med. 2013;369:122-33. doi:10.1056/NEJMoa1302369.

11. Tumeh PC, Harview CL, Yearley JH, Shintaku IP, Taylor EJ, Robert L, et al. PD-1 blockade induces responses by inhibiting adaptive immune resistance. Nature. 2014;515:568-71. doi:10.1038/nature13954.

12. Herbst RS, Soria JC, Kowanetz M, Fine GD, Hamid O, Gordon MS, et al. Predictive correlates of response to the anti-PD-L1 antibody MPDL3280A in cancer patients. Nature. 2014;515:563-7. doi:10.1038/nature14011.

13. Steidl C, Connors JM, Gascoyne RD. Molecular pathogenesis of Hodgkin's lymphoma: increasing evidence of the importance of the microenvironment. J Clin Oncol. 2011;29:1812-26. doi:10.1200/JCO.2010.32.8401.

14. Ansell SM, Lesokhin AM, Borrello I, Halwani A, Scott EC, Gutierrez M, et al. PD-1 blockade with nivolumab in relapsed or refractory Hodgkin's Lymphoma. N Engl J Med. 2014. doi:10.1056/NEJMoa1411087.

15. Monti S, Savage KJ, Kutok JL, Feuerhake F, Kurtin P, Mihm M, et al. Molecular profiling of diffuse large B-cell lymphoma identifies robust subtypes including one characterized by host inflammatory response. Blood. 2005;105:1851-61.

16. Lenz G, Wright G, Dave SS, Xiao W, Powell J, Zhao H, et al. Stromal gene signatures in large-B-cell lymphomas. N Engl J Med. 2008;359:2313-23. doi:10.1056/NEJMoa0802885

17. Compagno M, Lim WK, Grunn A, Nandula SV, Brahmachary M, Shen Q, et al. Mutations of multiple genes cause deregulation of NF-kappaB in diffuse large B-cell lymphoma. Nature. 2009;459:717-21. doi:10.1038/nature07968.

18. Williams PM, Li R, Johnson NA, Wright G, Heath JD, Gascoyne RD. A novel method of amplification of FFPET-derived RNA enables accurate disease classification with microarrays. J Mol Diagn. 2010;12:680-6. doi:10.2353/ jmoldx.2010.090164.

19. Jima DD, Zhang J, Jacobs C, Richards KL, Dunphy CH, Choi WW, et al. Deep sequencing of the small RNA transcriptome of normal and malignant human B cells identifies hundreds of novel microRNAs. Blood. 2010;116:e118-27. doi:10.1182/blood-2010-05-285403.

20. Salaverria I, Philipp C, Oschlies I, Kohler CW, Kreuz M, Szczepanowski M, et al. Translocations activating IRF4 identify a subtype of germinal centerderived B-cell lymphoma affecting predominantly children and young adults. Blood. 2011;118:139-47. doi:10.1182/blood-2011-01-330795.

21. Hummel M, Bentink S, Berger H, Klapper W, Wessendorf S, Barth TF, et al. A biologic definition of Burkitt's lymphoma from transcriptional and genomic profiling. N Engl J Med. 2006;354:2419-30. doi:10.1056/NEJMoa055351.

22. Monti S, Chapuy B, Takeyama K, Rodig SJ, Hao Y, Yeda KT, et al. Integrative analysis reveals an outcome-associated and targetable pattern of p53 and cell cycle deregulation in diffuse large B cell lymphoma. Cancer Cell. 2012;22:359-72. doi:10.1016/j.ccr.2012.07.014.

23. Visco C, Li Y, Xu-Monette ZY, Miranda RN, Green TM, Tzankov A, et al. Comprehensive gene expression profiling and immunohistochemical studies support application of immunophenotypic algorithm for molecular subtype classification in diffuse large B-cell lymphoma: a report from the International DLBCL Rituximab-CHOP Consortium Program Study. Leukemia. 2012;26:2103-13. doi:10.1038/leu.2012.83.

24. Wright G, Tan B, Rosenwald A, Hurt EH, Wiestner A, Staudt LM. A gene expression-based method to diagnose clinically distinct subgroups of diffuse large B cell lymphoma. Proc Natl Acad Sci U S A. 2003;100:9991-6. 
25. Barrans SL, Crouch S, Care MA, Worrillow L, Smith A, Patmore R, et al. Whole genome expression profiling based on paraffin embedded tissue can be used to classify diffuse large B-cell lymphoma and predict clinical outcome. Br J Haematol. 2012;159:441-53. doi:10.1111/bjh.12045.

26. Alizadeh AA, Eisen MB, Davis RE, Ma C, Lossos IS, Rosenwald A, et al. Distinct types of diffuse large B-cell lymphoma identified by gene expression profiling. Nature. 2000;403:503-11.

27. Bea S, Zettl A, Wright G, Salaverria I, Jehn P, Moreno V, et al. Diffuse large B-cell lymphoma subgroups have distinct genetic profiles that influence tumor biology and improve gene-expression-based survival prediction. Blood. 2005;106:3183-90. doi:10.1182/blood-2005-04-1399.

28. Lenz G, Wright GW, Emre NC, Kohlhammer H, Dave SS, Davis RE, et al. Molecular subtypes of diffuse large B-cell lymphoma arise by distinct genetic pathways. Proc Natl Acad Sci U S A. 2008;105:13520-5. doi:10.1073/pnas.0804295105.

29. Barrett T, Troup DB, Wilhite SE, Ledoux P, Evangelista C, Kim IF, et al. NCBI GEO: archive for functional genomics data sets-10 years on. Nucleic Acids Res. 2011;39:D1005-10. doi:10.1093/nar/gkq1184.

30. Gray KA, Yates B, Seal RL, Wright MW, Bruford EA. Genenames.org: the HGNC resources in 2015. Nucleic Acids Res. 2014; doi:10.1093/nar/gku1071.

31. Wettenhall JM, Smyth GK. limmaGUl: a graphical user interface for linear modeling of microarray data. Bioinformatics. 2004;20:3705-6. doi:10.1093/ bioinformatics/bth449.

32. Care MA, Barrans S, Worrillow L, Jack A, Westhead DR, Tooze RM. A microarray platform-independent classification tool for cell of origin class allows comparative analysis of gene expression in diffuse large B-cell lymphoma. PLoS One. 2013;8, e55895. doi:10.1371/journal.pone.0055895.

33. Wordle ${ }^{\mathrm{TM}}$. http://www.wordle.net/.

34. Signature database. http://lymphochip.nih.gov/signaturedb/.

35. The Molecular Signatures Database (MSigDB). http://www.broadinstitute. org/gsea/msigdb/index.jsp.

36. GeneSigDB. http://compbio.dfci.harvard.edu/genesigdb/.

37. Shaffer AL, Wright G, Yang L, Powell J, Ngo V, Lamy L, et al. A library of gene expression signatures to illuminate normal and pathological lymphoid biology. Immunol Rev. 2006;210:67-85. doi:10.1111/j.0105-2896.2006.00373.x.

38. Subramanian A, Tamayo P, Mootha VK, Mukherjee S, Ebert BL, Gillette MA, et al. Gene set enrichment analysis: a knowledge-based approach for interpreting genome-wide expression profiles. Proc Natl Acad Sci U S A. 2005;102:15545-50.

39. Culhane AC, Schwarzl T, Sultana R, Picard KC, Picard SC, Lu TH, et al. GeneSigDB-a curated database of gene expression signatures. Nucleic Acids Res. 2010;38:D716-25. doi:10.1093/nar/gkp1015.

40. Rosenwald A, Wright G, Leroy K, Yu X, Gaulard P, Gascoyne RD, et al. Molecular diagnosis of primary mediastinal $B$ cell lymphoma identifies a clinically favorable subgroup of diffuse large B cell lymphoma related to Hodgkin lymphoma. J Exp Med. 2003;198:851-62. doi:10.1084/jem.20031074.

41. Maere S, Heymans K, Kuiper M. BiNGO: a Cytoscape plugin to assess overrepresentation of gene ontology categories in biological networks. Bioinformatics. 2005;21:3448-9. doi:10.1093/bioinformatics/bti551.

42. Gene Ontology Consortium. http://www.geneontology.org.

43. GENE-E. http://www.broadinstitute.org/cancer/software/GENE-E/.

44. Johnson PW, Davies A, Jack III A. Applying molecular phenotyping in practice. Hematol Oncol. 2013;31:29-32. doi:10.1002/hon.2063.

45. Sallusto F, Lenig D, Mackay CR, Lanzavecchia A. Flexible programs of chemokine receptor expression on human polarized T helper 1 and 2 lymphocytes. J Exp Med. 1998;187:875-83.

46. Kim CH, Kunkel EJ, Boisvert J, Johnston B, Campbell JJ, Genovese MC, et al. Bonzo/CXCR6 expression defines type 1-polarized T-cell subsets with extralymphoid tissue homing potential. J Clin Invest. 2001;107:595-601. doi:10.1172/JCl11902

47. Steidl C, Gascoyne RD. The molecular pathogenesis of primary mediastinal large B-cell lymphoma. Blood. 2011;118:2659-69. doi:10.1182/blood-201105-326538.

48. Challa-Malladi M, Lieu YK, Califano O, Holmes AB, Bhagat G, Murty W, et al. Combined genetic inactivation of beta2-Microglobulin and CD58 reveals frequent escape from immune recognition in diffuse large B cell lymphoma. Cancer Cell. 2011:20:728-40. doi:10.1016/j.ccr.2011.11.006.

49. Scott DW, Gascoyne RD. The tumour microenvironment in B cell lymphomas. Nat Rev Cancer. 2014;14:517-34. doi:10.1038/nrc3774.

50. Ahearne MJ, Bhuller K, Hew R, Ibrahim H, Naresh K, Wagner SD. Expression of PD-1 (CD279) and FoxP3 in diffuse large B-cell lymphoma. Virchows Arch. 2014;465:351-8. doi:10.1007/s00428-014-1615-5.
51. Chen BJ, Chapuy B, Ouyang J, Sun HH, Roemer MG, Xu ML, et al. PD-L1 expression is characteristic of a subset of aggressive B-cell lymphomas and virus-associated malignancies. Clin Cancer Res. 2013;19:3462-73. doi:10.1158/1078-0432.CCR-13-0855.

52. Chetaille B, Bertucci F, Finetti P, Esterni B, Stamatoullas A, Picquenot JM, et al. Molecular profiling of classical Hodgkin lymphoma tissues uncovers variations in the tumor microenvironment and correlations with EBV infection and outcome. Blood. 2009;113:2765-3775. doi:10.1182/blood2008-07-168096.

53. Van Loo P, Tousseyn T, Vanhentenrijk V, Dierickx D, Malecka A, Vanden Bempt I, et al. T-cell/histiocyte-rich large B-cell lymphoma shows transcriptional features suggestive of a tolerogenic host immune response. Haematologica. 2010;95:440-8. doi:10.3324/haematol.2009.009647.

54. Ninomiya S, Hara T, Tsurumi H, Hoshi M, Kanemura N, Goto N, et al. Indoleamine 2,3-dioxygenase in tumor tissue indicates prognosis in patients with diffuse large B-cell lymphoma treated with R-CHOP. Ann Hematol. 2011:90:409-16. doi:10.1007/s00277-010-1093-z.

55. Galon J, Costes A, Sanchez-Cabo F, Kirilovsky A, Mlecnik B, Lagorce-Pages C, et al. Type, density, and location of immune cells within human colorectal tumors predict clinical outcome. Science. 2006;313:1960-4. doi:10.1126/ science 1129139

56. Curtis C, Shah SP, Chin SF, Turashvili G, Rueda OM, Dunning MJ, et al. The genomic and transcriptomic architecture of 2,000 breast tumours reveals novel subgroups. Nature. 2012;486:346-52. doi:10.1038/nature10983.

57. Chew V, Chen J, Lee D, Loh E, Lee J, Lim KH, et al. Chemokine-driven lymphocyte infiltration: an early intratumoural event determining long-term survival in resectable hepatocellular carcinoma. Gut. 2012;61:427-38. doi:10.1136/gutjnl-2011-300509.

58. Ji RR, Chasalow SD, Wang L, Hamid O, Schmidt H, Cogswell J, et al. An immune-active tumor microenvironment favors clinical response to ipilimumab. Cancer Immunol Immunother. 2012;61:1019-31. doi:10.1007/ s00262-011-1172-6.

59. Ulloa-Montoya F, Louahed J, Dizier B, Gruselle O, Spiessens B, Lehmann FF et al. Predictive gene signature in MAGE-A3 antigen-specific cancer immunotherapy. J Clin Oncol. 2013;31:2388-95. doi:10.1200/ JCO.2012.44.3762.

60. Ansell SM, Hurvitz SA, Koenig PA, LaPlant BR, Kabat BF, Fernando D, et al Phase I study of ipilimumab, an anti-CTLA-4 monoclonal antibody, in patients with relapsed and refractory B-cell non-Hodgkin lymphoma. Clin Cancer Res. 2009;15:6446-53. doi:10.1158/1078-0432.CCR-09-1339.

61. Armand P, Nagler A, Weller EA, Devine SM, Avigan DE, Chen YB, et al. Disabling immune tolerance by programmed death-1 blockade with pidilizumab after autologous hematopoietic stem-cell transplantation for diffuse large B-cell lymphoma: results of an international phase II trial. J Clin Oncol. 2013;31:4199-206. doi:10.1200/JCO.2012.48.3685.

62. Powles T, Eder JP, Fine GD, Braiteh FS, Loriot Y, Cruz C, et al. MPDL3280A (anti-PD-L1) treatment leads to clinical activity in metastatic bladder cancer. Nature. 2014;515:558-62. doi:10.1038/nature13904.

63. Ali K, Soond DR, Pineiro R, Hagemann T, Pearce W, Lim EL, et al. Inactivation of $\mathrm{PI}(3) \mathrm{K}$ p110delta breaks regulatory T-cell-mediated immune tolerance to cancer. Nature. 2014;510:407-11. doi:10.1038/nature13444.

64. Clark DA, Chaouat G, Arck PC, Mittruecker HW, Levy GA. Cytokinedependent abortion in CBA $\times$ DBA/2 mice is mediated by the procoagulant fgl2 prothrombinase [correction of prothombinase]. J Immunol. 1998;160:545-9.

65. Parr RL, Fung L, Reneker J, Myers-Mason N, Leibowitz JL, Levy G. Association of mouse fibrinogen-like protein with murine hepatitis virus-induced prothrombinase activity. J Virol. 1995;69:5033-8.

66. Chan CW, Kay LS, Khadaroo RG, Chan MW, Lakatoo S, Young KJ, et al. Soluble fibrinogen-like protein 2/fibroleukin exhibits immunosuppressive properties: suppressing $T$ cell proliferation and inhibiting maturation of bone marrow-derived dendritic cells. J Immunol. 2003;170:4036-44.

67. Shalev I, Liu H, Koscik C, Bartczak A, Javadi M, Wong KM, et al. Targeted deletion of fgl 2 leads to impaired regulatory $T$ cell activity and development of autoimmune glomerulonephritis. J Immunol. 2008;180:249-60.

68. Joller N, Lozano E, Burkett PR, Patel B, Xiao S, Zhu C, et al. Treg cells expressing the coinhibitory molecule TIGIT selectively inhibit proinflammatory Th1 and Th17 cell responses. Immunity. 2014;40:569-81. doi:10.1016/j.immuni.2014.02.012

69. Khattar R, Luft O, Yavorska N, Shalev I, Phillips MJ, Adeyi O, et al. Targeted deletion of FGL2 leads to increased early viral replication and enhanced 
adaptive immunity in a murine model of acute viral hepatitis caused by LCMV WE. PLoS One. 2013;8, e72309. doi:10.1371/journal.pone.0072309.

70. Marazzi S, Blum S, Hartmann R, Gundersen D, Schreyer M, Argraves S, et al. Characterization of human fibroleukin, a fibrinogen-like protein secreted by T lymphocytes. J Immunol. 1998;161:138-47.

71. Lafuse WP, Castle L, Brown D, Zwilling BS. The cytotoxic T Iymphocyte gene FIBLP with homology to fibrinogen beta and gamma subunits is also induced in mouse macrophages by IFN-gamma. Cell Immunol. 1995;163:187-90. doi:10.1006/cimm.1995.1115.

72. Shalev I, Wong KM, Foerster K, Zhu Y, Chan C, Maknojia A, et al. The novel $\mathrm{CD} 4+\mathrm{CD} 25+$ regulatory $T$ cell effector molecule fibrinogen-like protein 2 contributes to the outcome of murine fulminant viral hepatitis. Hepatology. 2009:49:387-97. doi:10.1002/hep.22684.

73. Urbanellis P, Shyu W, Khattar R, Wang J, Zakharova A, He W, et al. The Treg effector molecule fibrinogen-like protein 2 is necessary for the development of rapamycin-induced tolerance to fully $\mathrm{MHC}$-mismatched murine cardiac allografts. Immunology. 2014; doi:10.1111/imm.12354.

74. Long HM, Taylor GS, Rickinson AB. Immune defence against EBV and EBVassociated disease. Curr Opin Immunol. 2011;23:258-64. doi:10.1016/ j.coi.2010.12.014

75. Macedo C, Webber SA, Donnenberg AD, Popescu I, Hua Y, Green M, et al. EBV-specific CD8+ T cells from asymptomatic pediatric thoracic transplant patients carrying chronic high EBV loads display contrasting features: activated phenotype and exhausted function. J Immunol. 2011;186:5854-62. doi:10.4049/jimmunol.1001024.

76. Morscio J, Dierickx D, Ferreiro JF, Herreman A, Van Loo P, Bittoun E, et al. Gene expression profiling reveals clear differences between EBV-positive and EBV-negative posttransplant lymphoproliferative disorders. Am J Transplant. 2013;13:1305-16. doi:10.1111/ajt.12196.

77. Park S, Lee J, Ko YH, Han A, Jun HJ, Lee SC, et al. The impact of Epstein-Barr virus status on clinical outcome in diffuse large B-cell lymphoma. Blood. 2007;110:972-8. doi:10.1182/blood-2007-01-067769.

\section{Submit your next manuscript to BioMed Central and take full advantage of:}

- Convenient online submission

- Thorough peer review

- No space constraints or color figure charges

- Immediate publication on acceptance

- Inclusion in PubMed, CAS, Scopus and Google Scholar

- Research which is freely available for redistribution 